\title{
Vertical transmission of Wolbachia in Tetranychus kanzawai Kishida and Panonychus mori Yokoyama (Acari: Tetranychidae)
}

\author{
X-Y Hong ${ }^{1,2}, \mathrm{~T}_{\text {Gotoh }}{ }^{1}$ and T Nagata ${ }^{1}$ \\ ${ }^{1}$ Laboratory of Applied Entomology and Zoology, Faculty of Agriculture, Ibaraki University, Ami, Ibaraki 300-0393, Japan; \\ ${ }^{2}$ Department of Entomology, School of Plant Protection, Nanjing Agricultural University, Jiangsu 210095, China
}

The vertical transmission of Wolbachia in two species of spider mite was investigated and compared. One species, Tetranychus kanzawai Kishida, was infected with a modification negative strain of Wolbachia while the other species, Panonychus mori Yokoyama, was infected with a modification positive strain. The infection showed perfect maternal transmission in the laboratory population of $T$. kanzawai in which Wolbachia-infected females produced infected offspring regardless of whether they mated with infected or uninfected males, and uninfected females produced Wolbachia-free progenies without regard to the infection status of their mating partners. In artificial $P$. mori popu- lations initiated with 50\% infected and 50\% uninfected female adults, the infection frequencies among progenies increased with each generation, reaching $100 \%$ at the sixth generation in the Sendai population and after the sixth generation in the Toyama population. In another experiment, in which an artificial $T$. kanzawai population was composed of $50 \%$ infected and $50 \%$ uninfected female adults, the infection frequency in progeny populations increased very slowly, reaching $62.5 \%$ at the 15 th generation. The difference in infection frequency in the two spider mites may be due to the different strains of Wolbachia.

Heredity (2002) 88, 190-196. DOI: 10.1038/sj/hdy/6800026

Keywords: Wolbachia; Tetranychus kanzawai Kishida; Panonychus mori Yokoyama; vertical transmission

\section{Introduction}

Wolbachia are maternally inherited alpha-proteobacteria known to infect a wide range of arthropods (Werren et al, 1995; Bouchon et al, 1998). Infections with Wolbachia have been associated with various reproductive abnormalities in the host, such as parthenogenesis, feminization and cytoplasmic incompatibility (CI) (Werren, 1997; Stouthamer et al, 1999). CI-causing infections are expected to spread within and among populations, because infected females who are 'immune' to CI will, on average, produce more adult progeny than uninfected females, and this spread can occur quickly if CI is intense (Turelli and Hoffmann, 1999). In natural Drosophila simulans populations in California, incompatible crosses between uninfected females and infected males produce, on average, only about half as many progeny as compatible crosses; the infection has spread throughout central and northern parts of the state within the last decade (Turelli and Hoffmann, 1991, 1995). A Wolbachia infection also appears to be spreading northeasterly among populations of the small brown planthopper, Laodelphax striatellus, in Japan (Hoshizaki and Shimada, 1995).

In Acari, Wolbachia have been found to induce CI in Tetranychus urticae Koch and Tetranychus turkestani (Ugarov and Nikolski) (Breeuwer, 1997; van Opijnen and

Correspondence: $T$ Gotoh, Laboratory of Applied Entomology and Zoology, Faculty of Agriculture, Ibaraki University, Ami, Ibaraki 3000393, Japan.E-mail:gotoh@msv.ipc.ibaraki.ac.jp

Received 12 June 2001; accepted 24 October 2001
Breeuwer, 1999), and in Metaseiulus occidentalis (Nesbitt) (Johanowicz and Hoy, 1998). On the other hand, Wolbachia have been confirmed not to be responsible for CI in Tetranychus kanzawai Kishida (Gomi et al, 1997) and T. urticae (Gotoh et al, 1999a) and between the $\mathrm{T}$ and $\mathrm{K}$ strains of T. kanzawai (Gotoh et al, 1999b), or for the thelytokous mode of reproduction in oribatid mites (PerrotMinnot and Norton, 1997). Therefore, the effect of Wolbachia on the reproduction of spider mites is still a subject of debate. Further studies of the mechanisms by which Wolbachia affects spider mites are clearly needed.

The non-reciprocal nature of cytoplasmic incompatibility indicates that the male's contribution is critical to CI expression. However, Wolbachia are not present in mature sperm; paternal transmission occurs only rarely, if at all (Hoffmann and Turelli, 1988). Wolbachia in adult males somehow render the sperm incapable of successfully completing fertilization after entry into an uninfected egg cytoplasm (O'Neill and Karr, 1990). Compatible crosses involving infected females produce normal progeny regardless of the infection state of the male. Therefore, eggs derived from infected females somehow negate or rescue the action of Wolbachia on sperm (Boyle et al, 1993).

Further research on the vertical transmission of Wolbachia by their hosts is needed to understand the cellular and molecular mechanisms of CI. This is because Wolbachia are vertically transmitted from mother to offspring via the egg cytoplasm; males do not transmit Wolbachia. To our knowledge, there have been no studies on the vertical transmission of Wolbachia in Acari hosts. The 
purpose of this study is two-fold. First, it will compare how two different strains of Wolbachia vertically transmit to the offspring generation. Second, it provides an insight into the mechanisms of vertical transmission of Wolbachia. For our study, we used T. kanzawai, an important spider mite pest in Japan and China (Ehara and Shinkaji, 1996; Zhang et al, 1996), and Panonychus mori Yokoyama, a pest on deciduous fruit trees in Japan (Ehara and Gotoh, 1992).

\section{Materials and methods}

\section{Mite species}

The kanzawa spider mite, T. kanzawai was collected from tea fields in the Haibara area $\left(34^{\circ} 46^{\prime} \mathrm{N}-138^{\circ} 13^{\prime} \mathrm{E}\right)$, Shizuoka Prefecture, Japan. The mites were reared on detached leaves of kidney bean (Phaseolus vulgaris L.) in the laboratory under a controlled environment $\left(25^{\circ} \mathrm{C}, 60\right.$ $70 \%$ R.H., 16L-8D). For the detailed methods, see Gotoh et al (1999b).

Panonychus mori was collected from mulberry fields in Toyama city $\left(36^{\circ} 40^{\prime} \mathrm{N}-137^{\circ} 08^{\prime} \mathrm{E}\right)$, Toyama Prefecture, and Sendai city $\left(38^{\circ} 13^{\prime} \mathrm{N}-140^{\circ} 49^{\prime} \mathrm{E}\right)$, Miyagi Prefecture, Japan, and maintained on leaf discs of the mulberry (Morus bombycis Koidz.) placed on a water-saturated polyurethane mat in a petri dish ( $9 \mathrm{~cm}$ diameter). All experiments were also carried out at $25^{\circ} \mathrm{C}, 60-70 \%$ R.H. and $16 \mathrm{~L}-8 \mathrm{D}$ photoperiod.

Both species were confirmed to be infected with Wolbachia. The T. kanzawai Haibara population harbours a modification negative strain of Wolbachia at an infection rate of $63 \%$ (an initial field investigation of 80 individuals, unpublished data), and the P. mori Toyama and Sendai populations harbour a modification positive strain of Wolbachia at an infection rate of $100 \%$ (unpublished data). These two species were selected in order to compare the vertical transmission of these two types of Wolbachia. The Toyama and Sendai P. mori populations differ from each other in that the Wolbachia in the Toyama population always cause CI while the Wolbachia in the Sendai population cause CI only when its males mate with the females from Toyama population and not when the males mate with the females from other populations (Gotoh, unpublished data). The reason for this is currently under another investigation.

\section{Preparation of infected and uninfected lines}

In order to cross infected and uninfected individuals, $100 \%$ infected and $100 \%$ uninfected lines are needed. One female from the teleiochrysalis stage was introduced onto a leaf disc, and was allowed to lay eggs without being crossed with males. The eggs were reared until adulthood (males). The male adults were used to backcross with the mother female. After the cross, the female adults were transferred to new leaf discs and were allowed to lay eggs for 3-5 days. The female was checked for Wolbachia infection. The eggs were separately reared on new leaf discs depending on the infection status of the mother female. The above process was continued for three generations until a $100 \%$ infected population and an $100 \%$ uninfected population were obtained.

\section{Antibiotic treatment of $P$. mori}

Tetracycline has previously been shown to be effective against Wolbachia in spider mites (Breeuwer, 1997; Gomi et al, 1997). The aim of tetracycline treatment of $P$. mori

was to obtain uninfected lines for use in the crossing experiments. Small leaf discs (ca. $1 \mathrm{~cm}^{2}$ ) from the mulberry were placed on a cotton bed soaked in tetracycline solution $(0.1 \%, \mathrm{w} / \mathrm{v})$ in petri dishes (9 $\mathrm{cm}$ in diameter), and kept for $24 \mathrm{~h}$ before they were used for rearing the newly hatched larvae. Distilled water was added daily to keep the cotton beds wet. The cotton and the leaf discs were replaced every 4 days (see also Gotoh et al, 1995). After the treatment, mites were checked for infection of Wolbachia during the following three to five generations. Adults that were found to be uninfected were allowed to mate.

\section{DNA extraction and PCR amplification}

PCR was conducted to detect a gene that is specific to Wolbachia, the wsp gene (Braig et al, 1998). DNA was extracted by homogenizing a single female adult in the $25 \mu$ l mixture of STE buffer $(100 \mathrm{mM} \mathrm{NaCl}, 10 \mathrm{mM}$ Tris$\mathrm{HCl}, 1 \mathrm{mM}$ EDTA, $\mathrm{pH} 8.0)$ and proteinase $\mathrm{K}(10 \mathrm{mg} / \mathrm{ml}$, 2 microliters) in a 0.5-ml Eppendorf tube. The mixture was incubated at $37^{\circ} \mathrm{C}$ for $30 \mathrm{~min}$ and then at $95^{\circ} \mathrm{C}$ for 5 min. The sample was centrifuged briefly, and used immediately for the PCR reaction or stored at $-20^{\circ} \mathrm{C}$ for later use.

All PCR reactions were run in $26.25 \mu \mathrm{l}$ of buffer using a TAKARA Taq kit (No. R001B; Takara Co Ltd, Japan): $16 \mu \mathrm{l} \mathrm{H} \mathrm{H}_{2} \mathrm{O}, 2.5 \mu \mathrm{l}, 10 \times$ buffer, $1.5 \mu \mathrm{l}$ dNTP $(143 \mu \mathrm{m}$ each), $0.25 \mu \mathrm{l}$ Taq (1.25 U), $2 \mu \mathrm{l}$ sample, and $2 \mu \mathrm{l}$ of primers (20 pmol each). The primers for the wsp gene were $5^{\prime}$-TGG TCC AAT AAG TGA TGA AGA AAC-3' and 5'-AAA AAT TAA ACG CTA CTC CA-3' (Braig et al, 1998). Reactions were cycled 40 times at $95^{\circ} \mathrm{C}$ for $30 \mathrm{~s}, 52^{\circ} \mathrm{C}$ for 30 $\mathrm{s}$ and $72^{\circ} \mathrm{C}$ for $2 \mathrm{~min}$. Reagent negative and positive controls were included in the reactions. The PCR products were electrophoresed in a $1.0 \%$ agarose gel in $\mathrm{TBE} / \mathrm{EtBr}$ for $40 \mathrm{~min}$ at $60 \mathrm{~mA}$, and then photographed on a UV transilluminator. The techniques used here are modifications of the methods reported by Johanowicz and Hoy (1996), and Gomi et al (1997).

\section{Cross experiments and infection rate among the F1 generation in $T$. kanzawai}

Four cross combinations were carried out: uninfected females were crossed with uninfected males, uninfected females were crossed with infected males, infected females were crossed with uninfected males, and infected females were crossed with infected males. A cross was initiated by placing a teleiochrysalis female and a male adult on the same leaf disc. The egg laying was investigated every day. The adult female was allowed to lay eggs for 3 days and then was transferred to a new leaf disc. This was repeated for at least 15 days. The eggs deposited during each 3-day period were reared until adulthood. Upon emergence, all the female adults were checked for infection of Wolbachia. In this way we obtained the infection rate of the progeny in each 3-day period.

\section{Infection rate evolution}

Fifteen Wolbachia-infected and 15 Wolbachia-uninfected female individuals were sampled from the independent infected and uninfected systems and placed on the same leaf disc and allowed to lay eggs for 3 days. The infection rate at this stage was $50 \%$. Five days after the emergence 
of the next generation of female adults, 40 females were randomly sampled to check the infection rate and another 30 female adults were randomly selected and transferred to new leaf discs. The second-generation females on the new leaf discs were reared and allowed to lay eggs for 3 days, which developed into the third generation. Forty females in the third generation were used for assaying the infection rate, and 30 females were transferred to other new leaf discs. Three independent lines (three replicates) were set up for each generation, 40 female adults being sample from each replicate making, in total, 120 samples per generation. P. mori was checked for 10 generations and T. kanzawai was checked for 15 generations. All experiments were conducted under $25^{\circ} \mathrm{C} / 16 \mathrm{~L}-8 \mathrm{D}$.

\section{Cross experiments between infected and uninfected individuals}

To investigate how Wolbachia affects fecundity and how it is vertically transmitted, we conducted cross experiments with infected and uninfected individuals. For T. kanzawai, an infected male was mated with an infected female, and an uninfected male was mated with an uninfected female. For P. mori, four kinds of crosses were made: Sendai males were crossed with Sendai females, tetracyclinetreated Sendai males were crossed with tetracyclinetreated Sendai females, Toyama males were crossed with Toyama females, and tetracycline-treated Toyama males were crossed with tetracycline-treated Toyama females. The numbers of the eggs laid in the first 5 egg-laying days, the hatchability of the eggs, the survival rate of immatures and the sex ratio were recorded. All experiments were conducted under $25^{\circ} \mathrm{C} / 16 \mathrm{~L}-8 \mathrm{D}$.

\section{Results}

Infection rate among the $\mathrm{F} 1$ generation in $T$. kanzawai After eggs deposited in each of several 3-day periods developed into adults, the females were checked for Wolbachia infection with PCR. All the F1 offspring from crosses between infected males and infected females, and from crosses between uninfected males and infected females, were infected with Wolbachia, regardless of the time the eggs were deposited. The lower confidence intervals ranged between $99.52 \%$ and $87.79 \%$ (Table 1). On the other hand, none of the F1 offspring from crosses between infected males and uninfected females or from crosses between uninfected males and uninfected females were infected with Wolbachia, regardless of the time the eggs were deposited. The upper confidence intervals were from $0.44 \%$ to $22.09 \%$ (Table 1 ). Our results also clearly show that the offspring of the infected females were infected with Wolbachia and those of uninfected females were not infected with Wolbachia regardless of the Wolbachia-infection status of the male partner, and the infection rate remained the same for all of the F1 generation. This further illustrates that the infection mechanism of Wolbachia transmission in T. kanzawai or spider mites is cytoplasmic infection. Therefore, maternal transmission is perfect.

\section{Infection dynamics of Wolbachia in successive generations of offspring of $P$. mori}

Previous studies demonstrated that $P$. mori harbour a modification positive Wolbachia strain (Gotoh et al, 1998). The evolution of infection with this Wolbachia strain in successive generations of offspring of $P$. mori was investigated by sampling 40 females from each replicate line in each generation (ie 120 females/generation). We continued to monitor the infection status of each generation of offspring after it reached a 100\% infection rate. Two local populations were used for this experiment.

For the Sendai population, the F1 generation was infected at 59.2\%, which was slightly higher than the infection rate of the parent generation (50\%; Figure 1). The infection rate of the $\mathrm{F} 2$ generation reached $75.8 \%$. The remaining generations were infected at rates above $90 \%$. The infection rate reached $100 \%$ at the $\mathrm{F} 6$ generation, and remained at $100 \%$ until the F10 generation (Figure 1). Comparing the Sendai observed data with the expected data (theoretical changes), only one significant difference was found between the observed and the expected infection rates at the F2 generation and no difference was found at other generations (Figure 1). Regarding the Toyama population, the infection rates of the F1-F4 generations were $49.2 \%, 57.5 \%, 88.3 \%$ and $94.2 \%$ respectively, all of which showed significant differences from the expected infection rates at the F1-F4 generations (Figure 1). The infection rate remained high at $98.3 \%$ from the F5 generation and reached $100 \%$ at the F8 generation. No difference was found between the observed and the expected infection rates at F5-F10 generations (Figure 1).

The infection rate of the Sendai population rose quickly in the first three generations, exceeding $90 \%$ by the F3 generation, and then rose more slowly, reaching $100 \%$ by the F6 generation. Similarly, the infection rate of the Toyama population rose to more than $90 \%$ by the F4 gener-

Table 1 Infection rates among the F1 progeny in Tetranychus kanzawai

\begin{tabular}{|c|c|c|c|c|c|c|}
\hline Cross type & $N^{\mathrm{a}}$ & Days 1-3 & Days 4-6 & Days 7-9 & Day $10-12$ & Days $13-15$ \\
\hline 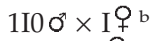 & 28 & $99.52 \%(619)^{c}$ & $99.44 \%(534)$ & $98.91 \%(274)$ & $97.36 \%(112)$ & $92.02 \%(36)$ \\
\hline UIó × I 9 & 27 & $99.42 \%(519)$ & $99.48 \%$ (577) & $97.59 \%(123)$ & $87.79 \%$ (23) & - \\
\hline $\mathrm{I}^{\prime \prime} \times \quad \mathrm{UI}+9$ & 23 & $0.76 \%(391)$ & $0.63 \%(472)$ & $0.94 \%(318)$ & $2.51 \%(118)$ & $22.09 \%(12)$ \\
\hline UIó × UI우 & 24 & $0.44 \%(680)$ & $0.47 \%(641)$ & $0.67 \%(446)$ & $2.37 \%(125)$ & $9.81 \%(29)$ \\
\hline
\end{tabular}

anumber of pairs crossed.

bI stands for infected, UI for uninfected.

'Numbers outside parenthesis are the lower confidence interval (I $\sigma^{\prime} \times$ I $q$ and UI $\sigma^{\prime \prime} \times$ I 9 ) or the upper confidence interval (I $\sigma^{\prime} \times$ UI $\$$ and UIO' $\times$ UIf); numbers inside parenthesis are PCR-investigated individuals. The lower confidence interval is calculated as N-th root of 0.05 $(0.05) \wedge(1 / \mathrm{n})$ for the crosses of Io $\times$ I 9 and UIO $\times$ I + , which have $100 \%$ transmission rate; and the upper confidence interval is calculated as $P=1-(0.05)^{\wedge}(1 / \mathrm{n})$ for the crosses of Io $\times$ UI 9 and UI $\sigma^{\prime \prime} \times$ UI $\$$, which have $0 \%$ transmission rate. 


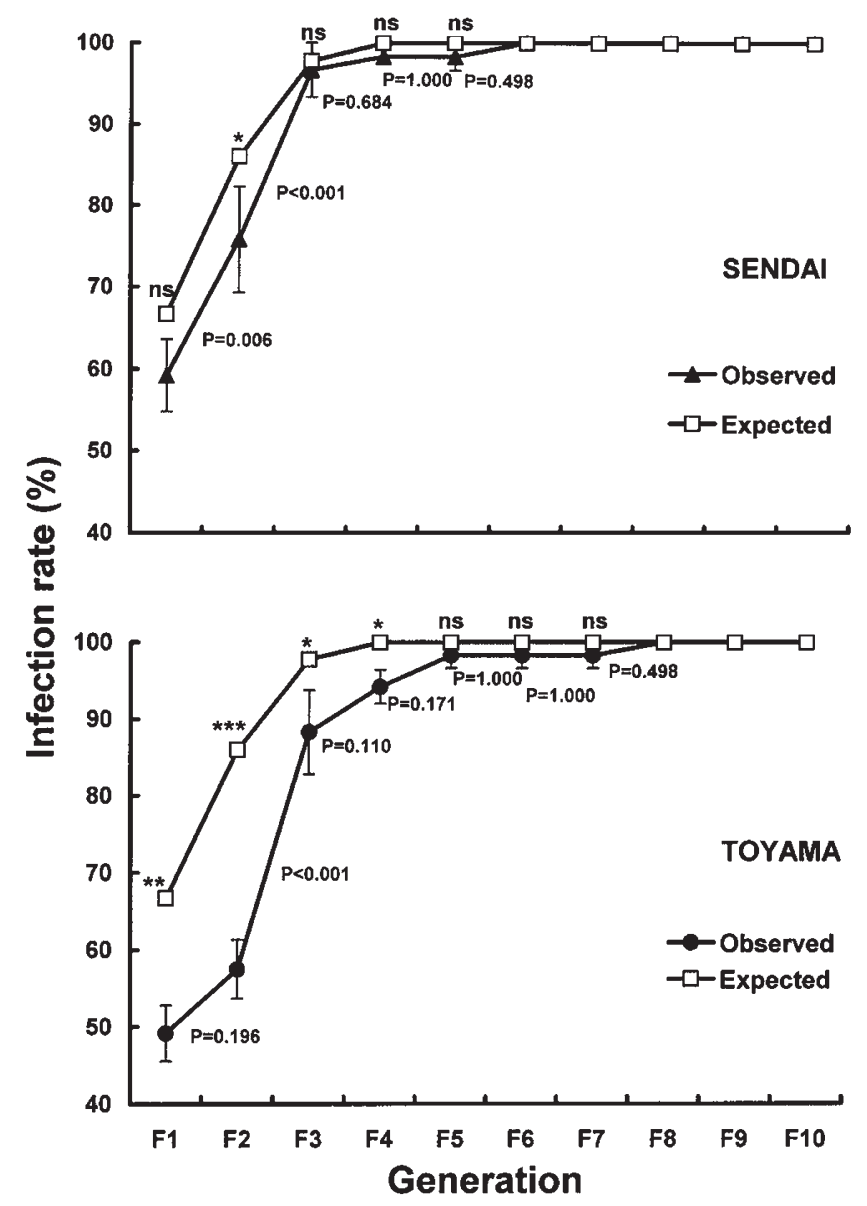

Figure 1 The infection rate of Wolbachia in the Sendai and Toyama populations of $P$. mori. For each generation, three replicates were set up and 40 female adults were sampled from each replicate to check Wolbachia infection. Bars indicate standard errors. P-value is the difference between two neighbouring generations, based on $\chi^{2}-$ test or Fisher's exact probability test. The expected infection rate change line was drawn up using the model of Hoffmann et al (1990). The tests between the observed and the expected infection rate at every generation were also done using $\chi^{2}$-test or Fisher's exact probability test. ns: $P>0.05,{ }^{*} P<0.05,{ }^{* *} P<0.01,{ }^{* * *} P<0.001$. The symbols were marked at the expected infection rate value.

ation, and then rose more slowly, reaching $100 \%$ by the F8 generation. No difference in infection rate was found between the Sendai and Toyama populations for any of the generation except $\mathrm{F} 2$, in which the infection rates were $75.8 \%$ and $57.5 \%$, respectively $\left(\chi^{2}=9.075, P=0.003\right.$; Figure 1).

\section{Infection dynamics of Wolbachia in successive} generations of offspring of $T$. kanzawai

Applying the same method used for $P$. mori populations, we monitored the infection dynamics of Wolbachia in $T$. kanzawai for 15 generations. The infection rates in the F1 to F4 generations were below $50 \%$. Starting at the F5 generation, the infection rate fluctuated around 55\% until it reached $60 \%$ at the F9 generation. Afterwards, the infection rate was between $60 \%$ and $67.5 \%$. These results indicate that the infection rate changed little with generations. No difference was found between the observed and the expected infection rates for each generation except those for the F10, F11 and F12 (Figure 2). The infection rates in the F10 to F12 generations were $63.3 \%$, $64.2 \%$ and $67.5 \%$, which were significantly higher than their respective expected infection rates.

The infection rate was $50 \%$ for the parent generation which consisted of 15 infected female adults and 15 uninfected female adults, but the infection rates of the generations F1 to F4 were below 50\%. The reason for the low infection rates in the first four generations with T. kanzawai is probably a consequence of the infection frequencies drifting, given the fairly small population size.

Fecundity comparison of infected and uninfected lines of $P$. mori and T. kanzawai

To further explore the factors governing the infection rate, an experiment was designed to examine the fecundity of infected and uninfected lines of P. mori and $T$. kanzawai. In P. mori, the fecundity did not appear to be adversely affected by the infection. The mean number of eggs laid in the first 5 days was not significantly different between the infected line and the tetracycline-treated line. The hatchability, survival rate and sex ratio were also not significantly different between the two lines (Table 2). Similarly, no significant difference was found between the infected and uninfected lines of T. kanzawai with respect to the eggs laid in the first 5 days or the sex ratio. However, significant differences were found between these two lines with respect to hatchability and the survival rate in immatures (Table 2).

\section{Discussion}

\section{Infection dynamics of Wolbachia in P. mori}

The Sendai and Toyama populations of $P$. mori harbour a modification positive strain of Wolbachia, which causes reproductive incompatibility when infected males mate with uninfected females. The non-reciprocal incompatibility can translate into a selective advantage to infected females when both infected and uninfected individuals are present in a population (Caspari and Watson, 1959; Turelli and Hoffmann, 1991). This is because infected females can reproduce normally with any male they encounter, while uninfected females mated with infected males produce few or no progeny (Barr, 1980). Our data from laboratory lines of both populations of $P$. mori are in accord with this and suggest that the infection rate can reach $100 \%$ rather than be stuck to a certain level, as suggested by some models in which one stable point is zero, and the other point is less than that of fixation of the bacterium, and therefore both infected and uninfected individuals can coexist in a population, if maternal transmission is imperfect (Fine, 1978; Hoffmann et al, 1990).

Two earlier laboratory studies have shown the spread of Wolbachia in cages containing populations of D. simulans (Hoffmann et al, 1990; Sinkins et al, 1995). The infection rate of Wolbachia in an artificial population consisting of infected and uninfected $D$. simulans increased rapidly within five to 10 generations to approximately 80-95\% (Hoffmann et al, 1990). The spread of Wolbachia was also noted by Sinkins et al (1995) who microinjected Wolbachiainfected $D$. simulans with a new strain of Wolbachia and found that the frequency of double-infected individuals increased from an initial prevalence of $10 \%$ to over $90 \%$ within 12 generations. However, a similar study by 


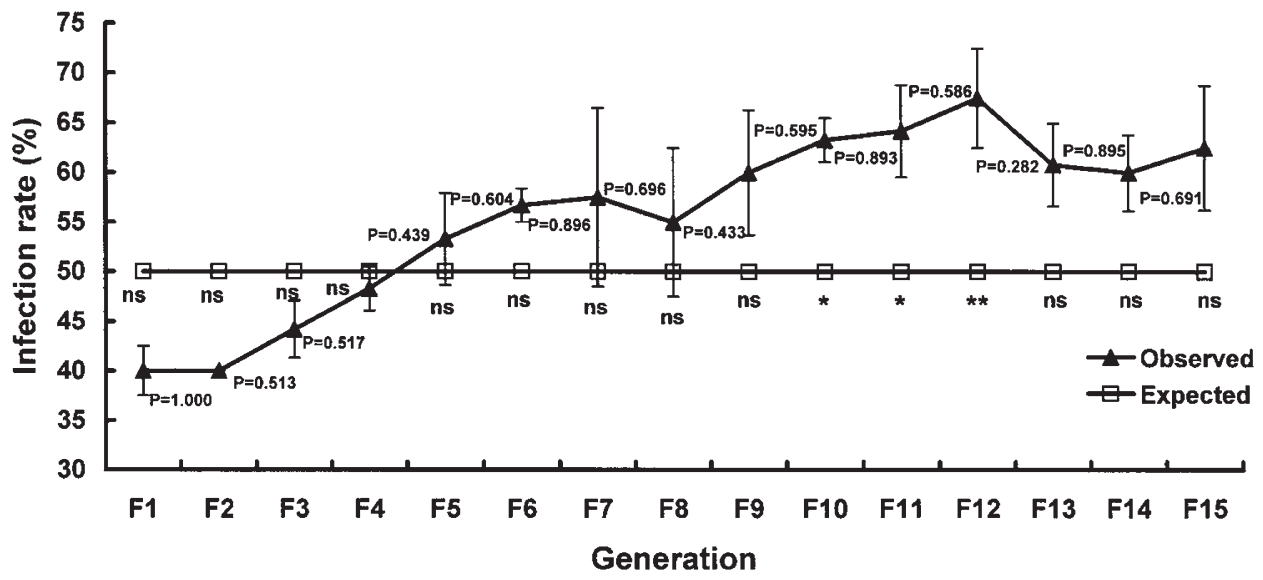

Figure 2 The infection rate of Wolbachia in T. kanzawai. For each generation, three replicates were set up and 40 female adults were sampled from each replicate to check Wolbachia infection. Bars indicate standard errors. P-value is the difference between two neighbouring generations, based on $\chi^{2}$-test or Fisher's exact probability test. The expected infection rate change line was drawn up using the model of Hoffmann et al (1996). The tests between the observed and the expected infection rate at every generation were also done using $\chi^{2}$-test or Fisher's exact probability test. ns: $P>0.05,{ }^{*} P<0.05,{ }^{* *} P<0.01$. The symbols were marked at the expected infection rate value.

Table 2 Results of crosses between infected and uninfected individuals in P. mori and T. kanzawai

\begin{tabular}{|c|c|c|c|c|c|c|}
\hline Species & Male $\times$ Female & $N^{\mathrm{a}}$ & $\begin{array}{l}\text { Eggs in } \\
\text { first } 5 \text { days }^{\mathrm{b}}\end{array}$ & $\begin{array}{c}\text { Hatchability } \\
(\%)^{\mathrm{b}}\end{array}$ & $\begin{array}{l}\text { Survival rate } \\
\text { in immatures }\end{array}$ & 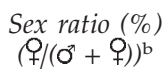 \\
\hline P. mori & $\begin{aligned} & \text { Sendai } \times \text { Sendai } \\
& \text { Sendai } \mathrm{T}^{\mathrm{c}} \times \text { Sendai } \mathrm{T} \\
& \mathrm{Z} \\
& \text { Toyama } \times \text { Toyama } \\
& \text { Toyama } \mathrm{T} \times \text { Toyama } \mathrm{T} \\
& \mathrm{Z}\end{aligned}$ & $\begin{array}{r}15 \\
10 \\
\\
9 \\
20\end{array}$ & $\begin{array}{c}31.7 \pm 3.13 \\
28.9 \pm 2.29 \\
-0.278^{\mathrm{NS}} \\
31.3 \pm 4.43 \\
31.9 \pm 1.97 \\
-0.118^{\mathrm{NS}}\end{array}$ & $\begin{array}{c}97.0 \pm 0.78 \\
95.9 \pm 1.02 \\
-0.959^{\mathrm{NS}} \\
98.1 \pm 1.08 \\
97.9 \pm 0.55 \\
-0.646^{\mathrm{NS}}\end{array}$ & $\begin{array}{c}96.0 \pm 1.14 \\
93.9 \pm 1.22 \\
-1.374^{\mathrm{NS}} \\
95.8 \pm 1.35 \\
96.5 \pm 0.73 \\
-0.095^{\mathrm{NS}}\end{array}$ & $\begin{array}{c}71.1 \pm 1.26 \\
70.0 \pm 2.27 \\
-0.139^{\mathrm{NS}} \\
70.6 \pm 2.97 \\
74.4 \pm 1.30 \\
-0.731^{\mathrm{NS}}\end{array}$ \\
\hline T. kanzawai & $\begin{array}{c}\mathrm{I} \times \mathrm{I}^{\mathrm{d}} \\
\mathrm{UI} \times \mathrm{UI}^{\mathrm{d}} \\
\mathrm{Z}\end{array}$ & $\begin{array}{l}20 \\
20\end{array}$ & $\begin{array}{c}45.0 \pm 2.10 \\
46.4 \pm 2.58 \\
-1.070^{\mathrm{NS}}\end{array}$ & $\begin{array}{c}93.3 \pm 0.72 \\
98.6 \pm 0.39 \\
-4.908^{* * *}\end{array}$ & $\begin{array}{c}91.6 \pm 0.92 \\
96.7 \pm 0.85 \\
-3.626^{* * *}\end{array}$ & $\begin{array}{c}79.3 \pm 2.36 \\
81.9 \pm 1.96 \\
-0.866^{\mathrm{NS}}\end{array}$ \\
\hline
\end{tabular}

a Number of pairs crossed.

${ }^{b}$ Data are shown as mean \pm s.e. Means are significantly different: ${ }^{* * *} P<0.001$; ${ }^{N S}$ not significantly different (Mann-Whitney U-test).

${ }^{\mathrm{C}} \mathrm{T}$ stands for tetracycline-treated.

dI stands for infected, UI for uninfected.

Johanowicz and Hoy (1999) failed to find the same result. They used three populations initiated with $10 \%$ infected and $90 \%$ uninfected cured eggs of a predator mite M. occidentalis and monitored them for 12 generations. Wolbachia infection did not spread rapidly through the populations. Imperfect transmission rates and fitness costs were detected that could have prevented the rapid spread of Wolbachia. To explain why Wolbachia did not increase in frequency, Johanowicz and Hoy (1999) suggested that the initial infection frequency of $10 \%$ was below an unstable equilibrium frequency (= threshold frequency). Our own results are consistent with this interpretation. Turelli and Hoffmann (1991) showed that if an infection is initiated below this unstable frequency, Wolbachia may be prevented from spreading, and may actually decline to zero.

Several models have been proposed to predict the infection dynamics of CI-induced Wolbachia in insect populations (Caspari and Watson, 1959; Fine, 1978; Hoffmann and Turelli, 1988; Hoffmann et al, 1990; Turelli and Hoffmann, 1995). The model developed by Hoffmann et al (1990) states that $p_{t+1}$, the proportion of infected individuals in generation $t+1$, is given by:

$$
p_{t+1}=\frac{p_{t}(1-\mu)\left(1-s_{f}\right)}{1-s_{f} p_{t}-s_{h} p_{t}\left(1-p_{t}\right)-\mu_{s_{h}} p_{t}^{2}\left(1-s_{f}\right)}
$$

where $s_{f}=1-\mathrm{F} ; s_{h}=1-\mathrm{H}$; and $p_{t}$ is the proportion of infected individuals. $\mathrm{H}$ is the relative hatch rate from incompatible fertilizations, $\mathrm{F}$ is the relative fecundity of infected females, $\mu$ is the uninfected fraction of offspring from infected mothers, and $p_{t}$ is the frequency of infected adults in generation. Based on our results and assuming strong CI and perfect transmission in the case of P. mori in the present study, $\mu=0$ (perfect maternal transmission), $\mathrm{H}=0$ (the relative hatch rate from incompatible fertilizations), $s_{h}=1-\mathrm{H}=1, \mathrm{~F}=1$ (typically measured by comparing the average number of eggs laid per day by infected vs uninfected females, it is 1 if there is no significant difference in fecundity between infected and uninfected lines. Prof. Michael Turelli's personal communication, data from Table 2), $s_{f}=1-\mathrm{F}=0$, the model of Hoffmann et al (1990) predicts that the infection rates at different generations are $66.67 \%$ (F1), $86.02 \%$ (F2), $97.78 \%$ (F3), $99.96 \%$ (F4) and $100 \%$ (F5-F10). Our observed results for the Sendai population has shown a 
similar trend of infection rate change to that of these expected results, except for the F2 generation. However, in the Toyama population, the observed infection rates were significantly slower than the expected infection rates in the F1-F4 generations (Figure 1). It may be that differences in the Wolbachia strains in the two populations may be causing the differences between the observed and expected infection rate changes in the Sendai and Toyama populations.

We can think of four potential factors contributory to why the Wolbachia infection rate increased quickly in $P$. mori. First, the reproductive advantage of infected females theoretically acts rapidly to increase the prevalence of Wolbachia-infected hosts in a population (Caspari and Watson, 1959; Fine, 1978; Turelli and Hoffmann, 1991; Hoffmann et al, 1990). Our studies assumed that there was no relative fitness cost due to infection because the numbers of eggs deposited by the infected and uninfected individuals were not significantly different (Table 2). The model of Caspari and Waston (1959) predicts that without fitness costs, Wolbachia will readily spread through a population, even at low initial frequencies. Furthermore, our initial infection frequency was 50\%, which could add more chances for mating with infected individuals. Second, imperfect transmission rates (segregation) were not found during our PCR tests. This could be explained by the mating selection behaviour in spider mites. Dielman and Overmeer (1972) showed that when incompatible male spider mites ( $T$. urticae) were released into glasshouses, females preferred mating with compatible males rather than with incompatible males. Third, there were still chances for infected males to mate with uninfected females even though the infected males may have a preference for mating with infected females. The crosses between infected males and uninfected females resulted in CI (egg mortality) in P. mori. This resulted in a relatively lower number of offspring produced by uninfected females, and therefore it gradually reduced the number of uninfected individuals in the following generations. Fourth, P. mori has a low fecundity. The result from Sinkins et al (1995) showed that incompatibility levels were higher when a double-infected line of $D$. simulans was raised at a low density compared to a high density. The mites in the genus Panonychus lay fewer eggs than those in the genus Tetranychus.

\section{Infection dynamics of Wolbachia in T. kanzawai}

Contrary to the many models and much research on CI induced by Wolbachia in insects, only few studies have been carried out on the infection dynamics of Wolbachia that do not cause CI. The type A infections of $D$. simulans (Hoffmann et al, 1996) and Wolbachia infection in D. mauritiana (Giordano et al, 1995) among others, do not seem to lower hatching rates when infected males are mated to uninfected females. In the absence of incompatibility, Hoffmann et al (1996) proposed that the frequency of a Wolbachia infection $(p)$ in an isolated population at generation $p_{t+1}$ is given by the equation:

$$
p_{t+1}=\frac{p_{t}(1-\mu)\left(1-s_{f}\right)}{1-s_{h} p_{t}}
$$

where the parameters are as defined above. If $\mu$ is greater than 0 and/or $s_{f}$ falls between 0 and 1, then the infection will eventually be lost because $p_{t+1}$ will be less than $p_{t}$.

Our data clearly indicate that $\mu$ is 0 and maternal trans- mission is perfect in T. kanzawai. Furthermore, we did not see any effect of Wolbachia on fecundity. Using the parameter estimates of $\mu=0$ (perfect maternal transmission), $\mathrm{H}=1$ (no CI), $s_{h}=1-\mathrm{H}=0, \mathrm{~F}=1$ (data from Table 2), $s_{f}=1-\mathrm{F}=0$, the above model predicts that $p_{t+1}=p_{t}$, meaning that the infection will remains at a $50 \%$ rate. We have shown that the change in frequency of Wolbachia infection in T. kanzawai is quite stable and generally accords with the expected changes (Figure 2). How does the Wolbachia infection become stable in a laboratory population? It is easy to understand how CI-inducing Wolbachia infection has spread because it causes incompatibility when infected males mate with uninfected females. However, evolutionary changes in the genome of Wolbachia and in the genome of their hosts do not necessarily favour increasing levels of incompatibility. Instead, theoretical models (Turelli, 1994) show that infections associated with lower levels of incompatibility can be favoured when the deleterious effect they have on their host organism is reduced. Like the data obtained for $D$. simulans by Hoffmann et al (1996), our data may represent the outcome of such an evolutionary process. Giordano et al (1995) suggested three ways in which Wolbachia could invade and be maintained in a $D$. mauritiana population: (1) the neutral bacteria could 'hitchhike' into the population, (2) the bacteria could be beneficial to the host that carries them, and (3) the bacteria could have a close relationship with the spindle apparatus of nuclei during division. However, our results showed no significant difference in the number of eggs produced, suggesting that the bacteria have no detectable effect on fecundity in this species. Another possible reason is that selection pressures on the symbiont have favoured an attenuated form of the bacteria (Prout, 1994).

In future work, we will monitor the Wolbachia infection frequency under field conditions. It may well be the case that in the field the maternal transmission is different from that in the laboratory, and fitness effects may be evident in the field that were not apparent in the laboratory. Based on the present results, we expect to find associations between the infection frequency of Wolbachia and the density of mites, and between the transmission rate and the fecundity of mites. Some of the questions that we hope to answer are how the preference for infected individuals is determined in a combined population, and what is the threshold initial infection frequency for an artificial population that will allow it to continue to be infected by Wolbachia.

\section{Acknowledgements}

X-Y Hong was supported by a fellowship from the Ministry of Education, Science, Sports and Culture (MESSC) of Japan. We thank Dr Hiroaki Noda of the National Institute of Agrobiological Sciences of Japan and Dr Sugihiko Hoshizaki of the University of Tokyo for their helpful discussions, and Dr Yasuki Kitashima of Ibaraki University and Dr Ai-Nong Zhou of Georgetown University, USA, for help with statistical analyses. We are also grateful to two unknown reviewers for their constructive comments on the manuscript. This study was supported in part by Grants-in-Aid for Scientific Research (nos. 09306003, 09660040 and 12460019) from the MESSC of Japan. 


\section{References}

Barr AR (1980). Cytoplasmic incompatibility in natural populations of a mosquito, Culex pipiens. Nature 283: 71-72.

Bouchon D, Rigaud T, Juchault P (1998). Evidence for widespread Wolbachia infection in isopod crustacean: molecular identification and host feminization. Proc R Soc Lond Ser B 265: 1081-1090.

Boyle L, O'Neill SL, Robertson HM, Karr TL (1993). Interspecific and intraspecific horizontal transfer of Wolbachia in Drosophila. Science 260: 1796-1799.

Braig HR, Zhou W-G, Dobson SL, O'Neill SL (1998). Cloning and characterization of a gene encoding the major surface protein of the bacterial endosymbiont Wolbachia pipientis. J Bacteriol 180: 2373-2378.

Breeuwer JAJ (1997). Wolbachia and cytoplasmic incompatibility in the spider mites Tetranychus urticae and T. turkestani. Heredity 79; 41-47.

Caspari E, Watson GS (1959). On the evolutionary importance of cytoplasmic sterility in mosquitoes. Evolution 13: 568-570.

Dielman J, Overmeer WPJ (1972). Preferential mating hampering the possibility to apply a genetic control against a population of Tetranychus urticae Koch. Zeitschrift für Angewandte Entomologie 71: 156-161.

Ehara S, Gotoh T (1992). Descriptions of two Panonychus spider mites from Japan, with a key to species of the genus in the world (Acari: Tetranychidae). Appl Entomol Zool 27: 107-115.

Ehara S, Shinkaji N (1996). Principles of Plant Acarology. National Countryside Education Association: Tokyo, 419 pp (in Japanese).

Fine PEM (1978). On the dynamics of symbiont-dependent cytoplasmic incompatibility in culicine mosquitoes. J Invert Pathol 30: $10-18$.

Giordano R, O'Neill SL, Robertson HM (1995). Wolbachia infections and the expression of cytoplasmic incompatibility in Drosophila sechillia and D. mauritiana. Genetics 140: 1307-1317.

Gomi K, Gotoh T, Noda H (1997). Wolbachia having no effect on reproductive incompatibility in Tetranychus kanzawai Kishida (Acari: Tetranychidae). Appl Entomol Zool 32: 485-490.

Gotoh T, Gomi K, Fujita T, Noda H (1998). Wolbachia infection and reproductive incompatibility in Panonychus mori Yokoyama. In 10th International Congress of Acarology (Canberra), Abstract.

Gotoh T, Oku H, Moriya K, Odawara M (1995). Nucleus-cytoplasm causing reproductive incompatability between two populations of Tetranychus quercivorus Ehara et Gotoh (Acari: Tetranychidae). Heredity 74: 405-414.

Gotoh T, Sugasawa J, Nagata T (1999a). Reproductive compatibility of the two-spotted spider mite (Tetranychus urticae) infected with Wolbachia. Entomol Sci 2: 289-295.

Gotoh T, Gomi K, Nagata T (1999b). Incompatibility and host plant differences among populations of Tetranychus kanzawai Kishida (Acari: Tetranychidae). Appl Entomol Zool 34: 551-561.

Hoffmann AA, Turelli M (1988). Unidirectional incompatibility in Drosophila simulans: inheritance, geographic variation and fitness effects. Genetics 119: 435-444.

Hoffmann AA, Turelli M, Harshman LG (1990). Factors affecting the distribution of cytoplasmic incompatibility in Drosophila simulans. Genetics 126: 933-948.

Hoffmann AA, Clancy D, Duncan J (1996). Naturally-occurring Wolbachia infection in Drosophila simulans that does not cause cytoplasmic incompatibility. Heredity 76: 1-8.

Hoshizaki S, Shimada T (1995). PCR-based detection of Wolbachia, cytoplasmic incompatibility microorganisms, infected in natural populations of Laodelphax striatellus (Homoptera: Delphacidae) in central Japan: has the distribution of Wolbachia spread recently? Insect Mol Biol 4: 237-243.

Johanowicz DL, Hoy MA (1996). Wolbachia in a predator-prey system: 16S ribosomal DNA analysis of two phytoseiids (Acari: Phytoseiidae) and their prey. Ann Entomol Soc Am 89: 435-441.

Johanowicz DL, Hoy MA (1998). Experimental introduction and termination of non-reciprocal reproductive incompatibilities in a parahaploid mite. Entomol Exp Appl 87: 51-58.

Johanowicz DL, Hoy MA (1999). Wolbachia infection dynamics in experimental laboratory populations of Metaseiulus occidentalis. Entomol Exp Appl 93: 259-268.

O'Neill SL, Karr TL (1990). Bidirectional incompatibility between conspecific populations of Drosophila simulans. Nature 348: 178 .

Perrot-Minnot M-J, Norton RA (1997). Obligate thelytoky in Oribatid mites: no evidence for Wolbachia inducement. Can Entomol 129: 691-698.

Prout T (1994). Some evolutionary possibilities for a microbe that causes incompatibility in its host. Evolution 48: 909-911.

Sinkins S, Braig HR, O'Neil SL (1995). Wolbachia superinfections and the expressions of cytoplasmic incompatibility. Proc $R$ Soc Lond Ser B 261: 325-330.

Southamer R, Breeuwer JAJ, Hurst GDD (1999). Wolbachia pipientis: microbial manipulator of arthropod reproduction. Ann Rev Microbiol 53: 71-102.

Turelli M (1994). Evolution of incompatibility-inducing microbes and their hosts. Evolution 48: 1500-1513.

Turelli M, Hoffmann AA (1991). Rapid spread of an inherited incompatibility factor in California Drosophila. Nature 353: 440-442.

Turelli M, Hoffmann AA (1995). Cytoplasmic incompatibility in Drosophila simulans: dynamics and parameter estimates from natural populations. Genetics 140: 1319-1338.

Turelli M, Hoffmann AA (1999). Microbe-induced cytoplasmic incompatibility as a mechanism for introducing transgenes into arthropod populations. Insect Mol Biol 8: 243-255.

van Opijnen T, Breeuwer JAJ (1999). High temperature eliminate Wolbachia, a cytoplasmic incompatibility inducing endosymbiont, from the two-spotted spider mite. Exp Appl Acarol 23: 871-881.

Werren JH (1997). Biology of Wolbachia. Ann Rev Entomol 42: 587-609.

Werren JH, Guo LR, Windsor DW (1995). Distribution of Wolbachia among neotropical arthropods. Proc R Soc Lond Ser B 262: 197-204.

Zhang YX, Yu D, Chen W, Chi YB, Lin JZ (1996). Study on the spatial distribution and temporal dynamics of Tetranychus kanzawai (Acari: Tetranychidae) in open-air strawberry gardens. Syst Appl Acarol 1: 73-76. 